\title{
An Introduction to this Special Issue: Chemosensation and Health
}

\author{
John E. Hayes ${ }^{1}$
}

Received: 14 September 2015 / Accepted: 15 September 2015 /Published online: 26 September 2015

(C) Springer Science+Business Media New York 2015

Relationships between the chemosenses, ingestive behaviors, food choice, and other health-related behaviors have long interested researchers across many fields (e.g., Fischer et al. 1963; Kang et al. 1967; Kaplan et al. 1964; Pangborn and Simone 1958). Indeed, rather than being "lower order" senses relative to vision and hearing, the chemosenses provided fertile soil for integrative, translational, transdisciplinary research (e.g., Barker 1982; Kare and Maller 1967) long before it was en vogue to speak of research in these terms. A prime example is the work by Kaplan and colleagues showing a link between genetic differences in taste perception and tobacco use (Kaplan et al. 1964). Other seminal work in this area includes a truly epic study by Pangborn and Simone from 1958: after collecting sweetness preferences in real foods from over 12, 000 individuals, they failed to find any relationship with body size (Pangborn and Simone 1958), suggesting the putative role of a sweet tooth in obesity may be a myth, albeit one that remains controversial today. Indeed, subsequent work on liking and intake has implicated fat rather than sweet foods in obesity risk (i.e., Drewnowski et al. 1985; Macdiarmid et al. 1998).

Given such a rich history, this special issue of Chemosensory Perception continues the exploration of connections between human chemosensation and health across a wide array of fields and, in doing so, highlights specific implications for chemosensory researchers, health care providers, as well as public health. Four of the articles in this

John E. Hayes

jeh40@psu.edu

1 Sensory Evaluation Center, Department of Food Science, College of Agricultural Sciences, The Pennsylvania State University, 220 Food Science Building, University Park, PA 16802, USA special issue are targeted reviews that cover a wide range of topics within the realm of chemosenses and health, while the remaining two articles are original research articles containing novel data.

Excessive sodium intake is a threat to public health in the developed world. The primary source of dietary sodium comes from processed foods themselves rather than being added at the table or during cooking (James et al. 1987; Mattes 1990). Accordingly, the US Institute of Medicine (IOM) released a guidance document (IOM 2010) 5 year ago that recommended gradual stepwise "stealth" reduction of the amount of sodium in foods as a means to reduce overall sodium intake, under the belief that consumer preferences would eventually shift to match reduced sodium levels in the diet. However, as Nuala Bobowoski highlights in her review (Bobowski 2015), this assumption has never been directly tested. Nuala's article succinctly reviews what is known about the ontogenty of salt preferences, the role of prior experience, and the gaps in current knowledge with regard to our ability to shift salt preferences over time.

The alterations of sensory function and shifts in food preferences that accompany cancer treatment are well known to anyone who has gone through treatment personally or watched a family member undergo treatment, at least anecdotally. Unfortunately, the training that oncologists and other allied health professionals receive in chemosensory biology and psychology is often very cursory, with the potential to influence the patient's will to withstand these necessary but challenging treatments. The second article in this issue is an expert commentary on chemosensation and sensory science within the context of cancer treatment (Boltong and Keast 2015). In this commentary, two experts in their respective fields-Anna Boltong and Russell Keast - open with the observation that health care providers are often confused by the terminology and jargon used by chemosensory researchers (e.g., taste 
versus flavor). Perhaps more critically, the authors also note this confusion is further compounded in clinical settings by a failure to distinguish true changes in sensory function from affective or hedonic shifts that are known to accompany cancer treatment. To address this confusion, they first provide clear definitions of core concepts, before presenting two theoretical frameworks to help clinicians conceptualize relevant issues. To help translate chemosensory knowledge to the clinic, they highlight the importance of patient self-report in conjunction with targeted questioning to help the clinician isolate the specific problem so that the patient's needs can be adequately addressed. They also emphasize the need for good anticipatory guidance from the clinician to help the patient successfully navigate the cancer treatment process.

The third article in this issue tackles a problem many of us, even those of us who work in the chemosenses every day, likely have not considered previously. Here, Donna Scarborough and Cathy Pelletier address the role of the chemosenses as they relate to swallowing, and swallowing disorders (Scarborough and Pelletier 2015). They first review the complex sequence of events that underlie swallowing, a process that most of us take for granted, before delving into changes across the lifespan. With respect to translation, they note chemesthetic stimuli like carbonation or concentrated citric acid appear to beneficial for swallowing safety in individuals with dysphagia; unfortunately, these stimuli are poorly tolerated by these individuals, limiting their utility. As noted by the authors, work in this area to date is relatively limited, with much still to be learned.

Pungent spices are widely and heavily consumed in many cuisines around the world, and interest in cuisines that contain these spices has steadily increased in western countries over the past two decades. Besides their important role in food flavor (Rozin 1983), it is now becoming apparent many of these spices may be bioactive in their own right (e.g., McCrea et al. 2015), with the potential to influence health and mortality (Lv et al. 2015). In the fourth article in this issue, Mary-Jon Ludy, Robin Tucker, and Sze-Yen Ten review the role of pungent spices in modulating food intake (Ludy et al. 2015). As is only appropriate given the scope of this issue, they limit themselves to work in humans that involve some degree of oral or nasal exposure, excluding trials where the spices are encapsulated or otherwise prevented from eliciting chemosensory responses. As pungent spices may potentially enhance or suppress intake, they cover both in their review, beginning with the former. Within each section, they provide detailed summaries that emphasize what is known, highlighting when this knowledge and clinical practice do not align.

Each year, the National Health and Nutrition Examination Survey (NHANES) is administered by the Centers for Disease Control and Prevention to a nationally representative sample of 5000 free-living individuals who range in age from birth to $80+$ years. This effort dates back to the first NHANES I study conducted between 1971 and 1975, with follow-up studies from 1976 to 1980 and 1988 to 1994 (NHANES II and III, respectively). Since 1999, NHANES shifted to a continuous design, where it provides a demographically weighted snapshot of the health and nutritional status of adults and children in the USA. The current NHANES study collects data via home-based interviews and a mobile exam facility staffed by trained medical personnel. The collected data includes demographic, socioeconomic, dietary, and health-related questions, as well as medical and dental exams, dietary interviews, and laboratory tests. After many years of effort spearheaded by Howard Hoffman and Barry Davis at the National Institutes of Deafness and Communications Disorders, with guidance from many other experts in the field, the NHANES team added a taste and smell exam component (http://www.cdc. gov/nchs/data/nhanes/nhanes_13_14/Taste_Smell.pdf) to the battery of tests conducted in the mobile examination center. In the fifth article in this special issue, Valerie Duffy and her team at UConn, in collaboration with Howard Hoffman, describe the results from a laboratory-based validation study of the NHANES taste and smell protocol in a convenience sample of adults (Rawal et al. 2015). They find that in spite of being a brief screening measure intended for epidemiological level data collection, the exam protocol both provides data that agrees with more extensive laboratory testing and has reasonable test-retest reliability over 6 months. As the newest wave of NHANES datasets become available to the public, the inclusion of valid, objective chemosensory tests can only help facilitate the exploration of new relationships between chemosenses, diet, and broader health.

The final article in this special issue contains original data from my laboratory at Penn State, with Alissa Allen Nolden as the lead author. Although the connections between the chemosenses and alcohol use, misuse, and abuse have long been studied (Kang et al. 1967; Mattes and DiMeglio 2001; Peeples 1962), we discovered a surprising gap in the literature when we went searching for reports on the change in perceptual quality of ethanol across concentrations that are ecologically relevant to alcoholic beverages. Here, in a convenience sample of adults who do not abstain from alcohol, we show that while sampled ethanol is simultaneously bitter, burning and sweet - as is readily apparent to anyone that has ever sipped neat vodka - the predominant sensation varies significantly across concentration, with bitterness dominating at lower concentrations, while burn dominates at higher concentrations (Nolden and Hayes 2015). Additionally, these data indicate that self-reported alcohol intake frequency outside the laboratory associates with individual differences in the sensation from straight ethanol (as opposed to alcoholic beverages, which also contain other sensory active components). This work contributes to a growing body of evidence that variation in chemosensation may differentially influence alcohol use 
(Dotson et al. 2012; Duffy et al. 2004; Hayes et al. 2011) and possibly misuse (Pelchat and Danowski 1992).

In closing, I would like to sincerely thank the journal editor in chief, Jeannine Delwiche, for giving me the opportunity to be the guest editor for this special issue, as this topic is very important to me. Also, I need to strongly thank the authors of all of the articles included here, as well as all the anonymous reviewers that reviewed these articles. I can only hope that this special issue stimulates additional interest in and work on the translation of the chemosenses out of the laboratory, into the clinic, and the public health arena.

Cheers!

John E. Hayes

Compliance with Ethics Requirements This article does not contain any studies with human participants or animals performed by any of the authors.

Conflict of Interest JEH has previously accepted speaking and/or consulting fees from industrial clients for unrelated projects, and he has also served on the Scientific Advisory Board of Medifast, Inc. His laboratory at Penn State also conducts routine taste tests for the food industry to facilitate experiential learning for undergraduate and graduate students. None of these organizations have had any influence over the conception, interpretation, or the decision to publish the information presented here.

\section{References}

Barker LM (1982) The psychobiology of human food selection. AVI Pub. Co, Westport

Bobowski N (2015) Shifting human salty taste preference: potential opportunities and challenges in reducing dietary salt intake of Americans. Chemosens Percept. doi:10.1007/s12078-015-9179-6

Boltong A, Keast R (2015) Chemosensory science in the context of cancer treatment: implications for patient care. Chemosen Percept. doi:10.1007/s12078-015-9180-0

Dotson CD, Wallace MR, Bartoshuk LM, Logan HL (2012) Variation in the gene TAS2R13 is associated with differences in alcohol consumption in patients with head and neck cancer. Chem Senses 37(8):737-44

Drewnowski A, Brunzell JD, Sande K, Iverius PH, Greenwood MR (1985) Sweet tooth reconsidered: taste responsiveness in human obesity. Physiol Behav 35(4):617-22

Duffy VB, Peterson J, Bartoshuk LM (2004) Associations between taste genetics, oral sensations and alcohol intake. Physiol Behav 82(2-3): 435-445

Fischer R, Griffin F, Kaplan AR (1963) Taste thresholds, cigarette smoking, and food dislikes. Med Exp Int J Exp Med 210:151-67

Hayes JE, Wallace MR, Knopik VS, Herbstman DM, Bartoshuk LM, Duffy VB (2011) Allelic variation in TAS2R bitter receptor genes associates with variation in sensations from and ingestive behaviors toward common bitter beverages in adults. Chem Senses 36(3):311-9

IOM (Institute of Medicine) (2010) Strategies to reduce sodium intake in the United States. National Academies Press, Washington DC. Available from: http://www.ncbi.nlm.nih.gov/books/NBK50956/. Accessed 4 Sept 2015

James WP, Ralph A, Sanchez-Castillo CP (1987) The dominance of salt in manufactured food in the sodium intake of affluent societies. Lancet 1(8530):426-9

Kang YS, Cho WK, Urn KS (1967) Taste sensitivity to phenylthiocarbamide of Korean population. Eugen Q 14(1):1-6

Kaplan AR, Glanville EV, Fischer R (1964) Taste thresholds for bitterness and cigarette smoking. Nature 202:1366

Kare MR, Maller O (1967) The chemical senses and nutrition. Johns Hopkins Press, Baltimore

Ludy M-J, Tucker RM, Tan S-Y (2015) Chemosensory properties of pungent spices: their role in altering nutrient intake. Chemosens Percept doi:10.1007/s12078-015-9191-x

Lv J, Qi L, Yu C, Yang L, Guo Y, Chen Y, Bian Z, Sun D, Du J, Ge P et al (2015) Consumption of spicy foods and total and cause specific mortality: population based cohort study. BMJ 351:h3942

Macdiarmid JI, Vail A, Cade JE, Blundell JE (1998) The sugar-fat relationship revisited: differences in consumption between men and women of varying BMI. Int J Obes Relat Metab Disord 22(11): 1053-61

Mattes RD (1990) Discretionary salt and compliance with reduced sodium diet. Nutr Res 10(12):1337-1352

Mattes RD, DiMeglio D (2001) Ethanol perception and ingestion. Physiol Behav 72(1-2):217-29

McCrea CE, West SG, Kris-Etherton PM, Lambert JD, Gaugler TL, Teeter DL, Sauder KA, Gu Y, Glisan SL, Skulas-Ray AC (2015) Effects of culinary spices and psychological stress on postprandial lipemia and lipase activity: results of a randomized crossover study and in vitro experiments. J Transl Med 13:7

Nolden AA, Hayes JE (2015) Perceptual qualities of ethanol depend on concentration, and variation in these percepts associates with drinking frequency. Chemosens Percept. doi:10.1007/s12078-0159196-5

Pangborn RM, Simone M (1958) Body size and sweetness preference. J Am Diet Assoc 34(9):924-8

Peeples EE. 1962. Taste sensitivity of phenylthiocarbamide in alcoholics [Masters thesis]. [DeLand, Florida]: Stetson University. p. 38.

Pelchat ML, Danowski S (1992) A possible genetic association between PROP-tasting and alcoholism. Physiol Behav 51(6):1261-6

Rawal S, Hoffman H, Honda M, Huedo-Medina T, Duffy V (2015) The taste and smell protocol in the 2011-2014 US National Health and Nutrition Examination Survey (NHANES): test-retest reliability and validity testing. Chemosens Percept. doi:10.1007/s12078-0159194-7

Rozin E (1983) Ethnic cuisine : the flavor-principle cook-book. Lexington, Mass.: S. Greene Press

Scarborough DR, Pelletier C (2015) The role of chemosenses in swallowing disorders across the lifespan. Chemosens Percept. doi:10.1007/s12078-015-9184-9 